International Journal of Difference Equations (IJDE).

ISSN 0973-6069, Volume 15, Number 2, (2020). 151-163

(C) Research India Publications

https://dx.doi.org/10.37622/IJDE/15.2.2020.151-163

\title{
Small Functions and Uniqueness of Difference Differential Polynomials of L-functions
}

\author{
Nintu Mandal ${ }^{1 *}$, and Nirmal Kumar Datta \\ Department of Mathematics, Chandernagore College, Chandernagore, \\ Hooghly-712136, West Bengal, India. \\ Department of Physics, Suri Vidyasagar College, Suri, Birbhum-731101, \\ West Bengal, India.
}

\begin{abstract}
In this paper, we study the value distributions of L-functions in the extended Selberg class. We prove two theorems which shows how difference differential polynomials of L-functions and difference differential polynomials of meromorphic functions uniquely determined concerning weighted sharing of small or rational functions. Our results improve and generalize some recent results due to W. J. Hao, J. F. Chen [3], W. Q. Zhu, J. F. Chen [16] and N. Mandal, N. K. Datta [10].
\end{abstract}

2010 Mathematics Subject Classification: 11M36, 30D35

Keywords: Meromorphic functions, L-functions, Weighted sharing, Uniqueness.

\section{INTRODUCTION}

L-functions are the most important objects in the modern number theory. The Riemann hypothesis and its extension to the general classes of L-functions is the most important unsolved problem in pure mathematics.

\footnotetext{
* Corresponding Author
} 
An L-function $L$ in the Selberg class is defined by the Dirichlet series $L(z)=$ $\sum_{n=1}^{\infty} a(n) / n^{z}$ which satisfies the following

(1) $a(n) \ll n^{\epsilon}$, for every $\epsilon>0$;

(2) There exists a nonnegative integer $k$ such that $(z-1)^{k} L(z)$ becomes an entire function of finite order;

(3) Every L-function satisfies the functional equation

$$
\lambda_{L}(z)=\omega \overline{\lambda_{L}(1-\bar{z})}
$$

where

$$
\lambda_{L}(z)=L(z) Q^{z} \prod_{i=1}^{k} \Gamma\left(\eta_{i} z+\nu_{i}\right)
$$

with positive real numbers $Q, \eta_{i}$ and complex numbers $\nu_{i}, \omega$ with $R e \nu_{i} \geq 0$ and $|\omega|=1$.

(4) $L(z)$ satisfies $L(z)=\prod_{p} L_{p}(z)$, where $L_{p}(z)=\exp \left(\sum_{k=1}^{\infty} b\left(p^{k}\right) / p^{k z}\right)$ with coefficients $b\left(p^{k}\right)$ satisfying $b\left(p^{k}\right) \ll p^{k \theta}$ for some $\theta<1 / 2$ and $p$ denotes prime number.

Clearly the Riemann zeta function is an L-function in the Selberg class. If $L$ satisfies (1) - (3) then we say that $L$ is an L-function in the extended Selberg class. In this paper by an L-function we mean an L-function in the extended Selberg class with $a(1)=1$.

In this paper, we study uniqueness problems with the help of Nevanlinna value distribution theory using the standard notations and definitions of the value distribution theory [4].

\section{PRELIMINARIES}

Let $\xi$ be a nonconstant meromorphic function. We denote by $S(r, \xi)$ any function satisfying $S(r, \xi)=o(T(r, \xi))$ as $r \longrightarrow \infty$, outside a possible exceptional set of finite linear measure. A meromorphic function $\rho$ is said to be a small function of $\xi$ if $T(r, \rho)=$ $S(r, \xi)$. The hyper order $\rho_{2}(\xi)$ of $\xi$ is defined by $\rho_{2}(\xi)=\limsup _{r \rightarrow \infty} \frac{\log \log T(r, \xi)}{\log r}$. Let $\alpha \in \mathbf{C} \cup\{\infty\}$ and $n$ be a positive integer. We denote by $E_{n)}(\alpha ; \xi)\left(\bar{E}_{n)}(\alpha ; \xi)\right)$ the set of all zeros of $\xi-\alpha$ with multiplicities not exceeding $n$, where zeros are counted according to their multiplicities(ignoring multiplicities).

In 2001 Lahiri [5, 6] introduced weighted sharing as follows

Definition 2.1. [5, 6]. Let $\xi$ and $\psi$ be meromorphic functions defined in the complex plane and $n$ be an integer $(\geq 0)$ or infinity. For $\alpha \in \mathbf{C} \cup\{\infty\}$ we denote by $E_{n}(\alpha ; \xi)$ 
the set of all zeros of $\xi-\alpha$ where a zero of multiplicity $k$ is counted $k$ times if $k \leq n$ and $n+1$ times if $k>n$. If $E_{n}(\alpha ; \xi)=E_{n}(\alpha ; \psi)$, we say that $\xi$, $\psi$ share the value $\alpha$ with weight $n$.

We write $\xi, \psi$ share $(\alpha, n)$ to mean that $\xi, \psi$ share the value $\alpha$ with weight $n$.

In 2017, Liu, Li and Yi [9] proved the following uniqueness theorems of L-functions.

Theorem 2.1. [9]. Let $j \geq 1$ and $k \geq 1$ be integers such that $j>3 k+6$. Also let $L$ be an $L$-function and $\xi$ be a nonconstant meromorphic function. If $\left\{\xi^{j}\right\}^{(k)}$ and $\left\{L^{j}\right\}^{(k)}$ share $(1, \infty)$ then $\xi \equiv \alpha L$ for some nonconstant $\alpha$ satisfying $\alpha^{j}=1$.

Theorem 2.2. [9]. Let $j \geq 1$ and $k \geq 1$ be integers such that $j>3 k+6$. Also let $L$ be an L-function and $\xi$ be a nonconstant meromorphic function. If $\left\{\xi^{j}\right\}^{(k)}(z)-(z)$ and $\left\{L^{j}\right\}^{(k)}(z)-(z)$ share $(0, \infty)$ then $\xi \equiv \alpha L$ for some nonconstant $\alpha$ satisfying $\alpha^{j}=1$.

Considering differential polynomials in 2018, W. J. Hao and J. F. Chen [3] obtain the following uniqueness results on L-function:

Theorem 2.3. [3]. Let $\xi$ be a nonconstant meromorphic function and $L$ be an L-function such that $\left[\xi^{n}(\xi-1)^{m}\right]^{(\tau)}$ and $\left[L^{n}(L-1)^{m}\right]^{(\tau)}$ share $(1, \infty)$, where $n, m, \tau \in$ $\mathbb{Z}^{+}$. If $n>m+3 \tau+6$ and $\tau \geq 2$, then, $\xi \equiv L$ or, $\xi^{n}(\xi-1)^{m} \equiv L^{n}(L-1)^{m}$.

Theorem 2.4. [3]. Let $\xi$ be a nonconstant meromorphic function and $L$ be an $L$-function such that $\left[\xi^{n}(\xi-1)^{m}\right]^{(\tau)}$ and $\left[L^{n}(L-1)^{m}\right]^{(\tau)}$ share $(1,0)$, where $n, m, \tau \in$ $\mathbb{Z}^{+}$. If $n>4 m+7 \tau+11$ and $\tau \geq 2$, then, $\xi \equiv L$ or, $\xi^{n}(\xi-1)^{m} \equiv L^{n}(L-1)^{m}$.

Using truncated sharing in 2019 W. Q. Zhu and J. F. Chen proved the following uniqueness theorem.

Theorem 2.5. [16]. Let $L$ be an L-function and $\xi$ be a transcendental meromorphic function defined in the complex plane $\boldsymbol{C}$. Also let $n, k(\geq 2), l(\geq 2)$ be positive integers such that $n \geq 7 k+17$. If $\bar{E}_{l)}\left(1,\left(\xi^{n}(\xi-1)\right)^{(k)}\right)=\bar{E}_{l)}\left(1,\left(L^{n}(L-1)\right)^{(k)}\right)$ then $\xi \equiv L$.

Definition 2.2. [8]. Let $\xi$ be a meromorphic function defined in the complex plane. Let $n$ be a positive integer and $\alpha \in \mathbf{C} \cup\{\infty\}$. By $N(r, \alpha ; \xi \mid \leq n)$ we denote the counting function of the $\alpha$ points of $\xi$ with multiplicity $\leq n$ and by $\bar{N}(r, \alpha ; \xi \mid \leq n)$ the reduced counting function. Also by $N(r, \alpha ; \xi \mid \geq n)$ we denote the counting function of the $\alpha$ points of $\xi$ with multiplicity $\geq n$ and by $\bar{N}(r, \alpha ; \xi \mid \geq n)$ the reduced counting function. We define

$$
N_{n}(r, \alpha ; \xi)=\bar{N}(r, \alpha ; \xi)+\bar{N}(r, \alpha ; \xi \mid \geq 2)+\cdots+\bar{N}(r, \alpha ; \xi \mid \geq n)
$$


Definition 2.3. [10]. Let $\xi, \chi$ be meromorphic functions defined in the complex plane and $\psi$ be a rational function or a small function of $\xi$ and $\chi$. Then we denote by $E_{m)}(\psi ; \xi), \bar{E}_{m)}(\psi ; \xi)$ and $E_{m}(\psi ; \xi)$ the sets $E_{m)}(0 ; \xi-\psi), \bar{E}_{m)}(0 ; \xi-\psi)$ and $E_{m}(0 ; \xi-\psi)$ respectively.

We write $\xi, \chi$ share $(\psi, n)$ to mean that $\xi-\psi, \chi-\psi$ share the value 0 with weight $n$. Clearly if $\xi, \chi$ share $(\psi, n)$ then $\xi, \chi$ share $(\psi, m)$ for all integers $m, 0 \leq m<n$. Also we note that $\xi, \chi$ share $\psi$ IM or CM if and only if $\xi$, $\chi$ share $(\psi, 0)$ or $(\psi, \infty)$ respectively.

Considering truncated sharing of small functions in 2020 Mandal and Datta [10] proved the following theorem.

Theorem 2.6. [10]. Let $L$ be a nonconstant $L$-function and $\rho$ be a small function of $L$ such that $\rho \not \equiv 0, \infty$. If $\bar{E}_{4)}(\rho ; L)=\bar{E}_{4)}\left(\rho ;\left(L^{m}\right)^{(k)}\right), E_{2)}(\rho ; L)=E_{2)}\left(\rho ;\left(L^{m}\right)^{(k)}\right)$ and

$$
2 N_{2+k}\left(r, 0 ; L^{m}\right) \leq(\sigma+o(1)) T(r, L),
$$

where $m \geq 1, k \geq 1$ are integers and $0<\sigma<1$, then $L \equiv\left(L^{m}\right)^{(k)}$.

Now the following questions comes naturally.

Question 2.1. If we take meromorphic function in place of trancendental meromorphic function in theorem 2.5 then what happens?

Question 2.2. Can we take difference differential polynomials in place of differential polynomials in theorem 2.3, 2.4, 2.5 and 2.6?

Definition 2.4. [8]. Let $\xi$ and $\psi$ be two meromorphic functions defined in the complex plane. Then we denote by $N(r, \psi ; \xi \mid \leq m), \bar{N}(r, \psi ; \xi \mid \leq m), N(r, \psi ; \xi \mid \geq m)$, $\bar{N}(r, \psi ; \xi \mid \geq m), N_{m}(r, \psi ; \xi)$ etc. the counting functions $N(r, 0 ; \xi-\psi \mid \leq m)$, $\bar{N}(r, 0 ; \xi-\psi \mid \leq m), N(r, 0 ; \xi-\psi \mid \geq m), \bar{N}(r, 0 ; \xi-\psi \mid \geq m), N_{m}(r, 0 ; \xi-\psi)$ etc. respectively.

Definition 2.5. [5]. Let two nonconstant meromorphic functions $\xi$ and $\psi$ share a value $(\alpha, 0)$. We denote by $\bar{N}_{*}(r, \alpha ; \xi, \psi)$ the counting function of the $\alpha$-points of $\xi$ and $\psi$ with different multiplicities, where each $\alpha$-point is counted only once.

Clearly $\bar{N}_{*}(r, \alpha ; \xi, \psi) \equiv \bar{N}_{*}(r, \alpha ; \psi, \xi)$. 
Definition 2.6. Let two nonconstant meromorphic functions $\xi$ and $\psi$ share a value $\alpha$ IM. We denote by $\bar{N}(r, \alpha ; \xi \mid>\psi)$ the counting function of the $\alpha$-points of $\xi$ and $\psi$ with multiplicities with respect to $\xi$ is greater than the multiplicities with respect to $\psi$, where each $\alpha$-point is counted once only.

Definition 2.7. Let two nonconstant meromorphic functions $\xi$ and $\psi$ share a value $\alpha$ IM. We denote by $\bar{N}_{E}(r, \alpha ; \xi, \psi \mid>m)$ the counting function of the $\alpha$-points of $\xi$ and $\psi$ with multiplicities greater than $m$ and the multiplicities with respect to $\xi$ is equal to the multiplicities with respect to $\psi$, where each $\alpha$-point is counted once only.

Definition 2.8. [7]. We denote by $N_{\otimes}\left(r, 0 ; \xi^{(k)}\right)\left(\bar{N}_{\otimes}\left(r, 0 ; \xi^{(k)}\right)\right)$ the counting function (reduced counting function) of those zeros of $\xi^{(k)}$ which are not the zeros of $\xi(\xi-1)$.

\section{MAIN RESULTS}

Using weighted sharing we try to solve Questions 2.1 and 2.2 and prove the following theorems.

Theorem 3.1. Let $L$ be a nonconstant $L$-function and $\xi$ be a meromorphic function. Let $\tau, n, \eta, \mu_{j}(j=1,2, \ldots, \eta), \lambda=\sum_{j=1}^{\eta} \mu_{j}$ be positive integers such that $n>$ $\lambda+(\eta+1)(3 \tau+4)$ and $\omega_{j} \in \boldsymbol{C}-\{0\}(j=1,2, \ldots, \eta)$ be distinct constants. Also let $\rho_{2}(L)<1, \rho_{2}(\xi)<1,\left[L^{n}(z) \prod_{j=1}^{\eta} L\left(z+\omega_{j}\right)^{\mu_{j}}\right]^{(\tau)}$ and $\left[\xi^{n}(z) \prod_{j=1}^{\eta} \xi\left(z+\omega_{j}\right)^{\mu_{j}}\right]^{(\tau)}$ share $(\rho(z), l)$ and $\xi, L$ share $(\infty, 0)$, where $2 \leq l<\infty$ and $\rho(z)$ is a small function of $\xi$ and $L$, then one of the following holds

(i) $\left[L(z)^{n} \prod_{j=1}^{\eta} L\left(z+\omega_{j}\right)^{\mu_{j}}\right]^{(\tau)} \equiv\left[\xi(z)^{n} \prod_{j=1}^{\eta} \xi\left(z+\omega_{j}\right)^{\mu_{j}}\right]^{(\tau)}$

(ii) $\left[L(z)^{n} \prod_{j=1}^{\eta} L\left(z+\omega_{j}\right)^{\mu_{j}}\right]^{(\tau)}\left[\xi(z)^{n} \prod_{j=1}^{\eta} \xi\left(z+\omega_{j}\right)^{\mu_{j}}\right]^{(\tau)} \equiv \rho(z)^{2}$.

Theorem 3.2. Let $L$ be a nonconstant $L$-function and $\xi$ be a meromorphic function. Let $\tau, n, \eta, \mu_{j}(j=1,2, \ldots, \eta), \lambda=\sum_{j=1}^{\eta} \mu_{j}$ be positive integers such that $n>$ $\lambda+(\eta+1)(3 \tau+4)$ and $\omega_{j} \in \boldsymbol{C}-\{0\}(j=1,2, \ldots, \eta)$ be distinct constants. Also let $\rho_{2}(L)<1, \rho_{2}(\xi)<1,\left[L^{n}(z) \prod_{j=1}^{\eta} L\left(z+\omega_{j}\right)^{\mu_{j}}\right]^{(\tau)}$ and $\left[\xi^{n}(z) \prod_{j=1}^{\eta} \xi\left(z+\omega_{j}\right)^{\mu_{j}}\right]^{(\tau)}$ share $(Q(z), l)$ and $\xi$, L share $(\infty, 0)$, where $2 \leq l<\infty$ and $Q(z)$ is a rational function, then one of the following holds

(i) $\left[L(z)^{n} \prod_{j=1}^{\eta} L\left(z+\omega_{j}\right)^{\mu_{j}}\right]^{(\tau)} \equiv\left[\xi(z)^{n} \prod_{j=1}^{\eta} \xi\left(z+\omega_{j}\right)^{\mu_{j}}\right]^{(\tau)}$ 
(ii) $\left[L(z)^{n} \prod_{j=1}^{\eta} L\left(z+\omega_{j}\right)^{\mu_{j}}\right]^{(\tau)}\left[\xi(z)^{n} \prod_{j=1}^{\eta} \xi\left(z+\omega_{j}\right)^{\mu_{j}}\right]^{(\tau)} \equiv Q(z)^{2}$.

\section{LEMMAS}

In this section we present some necessary lemmas.

Henceforth we denote by $\Omega$ the function defined by

$$
\Omega=\left(\frac{\Phi^{\prime \prime}}{\Phi^{\prime}}-\frac{2 \Phi^{\prime}}{\Phi-1}\right)-\left(\frac{\Psi^{\prime \prime}}{\Psi^{\prime}}-\frac{2 \Psi^{\prime}}{\Psi-1}\right)
$$

Lemma 4.1. [11]. Let $L$ be an L-function with degree q. Then

$$
T(r, L)=\frac{q}{\pi} r \log r+O(r) .
$$

Lemma 4.2. [10]. Let $L$ be an L-function. Then $N(r, \infty ; L)=S(r, L)=O(\log r)$.

Lemma 4.3. Let $\xi$ be a nonconstant meromorphic function and $L$ be an L-function. If $\xi$ and $L$ share $(\infty, 0)$ then $\bar{N}(r, \infty ; \xi)=S(r, L)=O(\log r)$.

Proof. Since $\xi$ and $L$ share $(\infty, 0)$ therefore by lemma 4.2 we have $\bar{N}(r, \infty ; \xi)=$ $\bar{N}(r, \infty ; L)=S(r, L)=O(\log r)$. This completes the proof.

Lemma 4.4. [15]. Let $\xi(z)=\frac{\alpha_{0}+\alpha_{1} z+\ldots .+\alpha_{n} z^{n}}{\beta_{0}+\beta_{1} z+\ldots .+\beta_{m} z^{m}}$ be a nonconstant rational function defined in the complex plane $\boldsymbol{C}$, where $\alpha_{0}, \alpha_{1}, \ldots ., \alpha_{n}(\neq 0)$ and $\beta_{0}, \beta_{1}, \ldots ., \beta_{m}(\neq 0)$ are complex constants. Then

$$
T(r, \xi)=\max \{m, n\} \log r+O(1) .
$$

Lemma 4.5. [12]. Let $\xi$ be a transcendental meromorphic function of hyper order $\rho_{2}(\xi)<1$. Then for any $\alpha \in \boldsymbol{C}-0$

$$
\begin{gathered}
T(r, \xi(z+\alpha))=T(r, \xi(z))+S(r, \xi(z)) \\
N(r, \infty ; \xi(z+\alpha))=N(r, \infty ; \xi(z))+S(r, \xi(z)) \\
N(r, 0 ; \xi(z+\alpha))=N(r, 0 ; \xi(z))+S(r, \xi(z))
\end{gathered}
$$

Lemma 4.6. [1]. Let $\Phi$ and $\Psi$ be two nonconstant meromorphic functions sharing $(1, l)$ and $(\infty, 0)$ where $2 \leq l<\infty$ and $\Omega \not \equiv 0$. Then

$$
\begin{aligned}
T(r, \Phi) & \leq N_{2}(r, 0 ; \Phi)+N_{2}(r, 0 ; \Psi)+\bar{N}(r, \infty ; \Phi)+\bar{N}(r, \infty ; \Psi)+\bar{N}_{*}(r, \infty ; \Phi, \Psi) \\
& -m(r, 1 ; \Psi)-N_{E}(r, 1 ; \Phi, \Psi \mid>3)-\bar{N}(r, 1 ; \Psi \mid>\Phi)+S(r, \Phi)+S(r, \Psi) \\
T(r, \Psi) & \leq N_{2}(r, 0 ; \Phi)+N_{2}(r, 0 ; \Psi)+\bar{N}(r, \infty ; \Phi)+\bar{N}(r, \infty ; \Psi)+\bar{N}_{*}(r, \infty ; \Phi, \Psi) \\
& -m(r, 1 ; \Phi)-N_{E}(r, 1 ; \Psi, \Phi \mid>3)-\bar{N}(r, 1 ; \Phi \mid>\Psi)+S(r, \Phi)+S(r, \Psi)
\end{aligned}
$$


Lemma 4.7. [14]. Let $\Phi$ be a nonconstant meromorphic function and $k, p$ be two positive integers. Then

$$
\begin{gathered}
T\left(r, \Phi^{(k)}\right) \leq T(r, \Phi)+k \bar{N}(r, \infty ; \Phi)+S(r, \Phi) \\
N_{p}\left(r, 0 ; \Phi^{(k)}\right) \leq T\left(r, \Phi^{(k)}\right)-T(r, \Phi)+N_{p+k}(r, 0 ; \Phi)+S(r, \Phi) \\
N_{p}\left(r, 0 ; \Phi^{(k)}\right) \leq N_{p+k}(r, 0 ; \Phi)+k \bar{N}(r, \infty ; \Phi)+S(r, \Phi) \\
N\left(r, 0 ; \Phi^{(k)}\right) \leq N(r, 0 ; \Phi)+k \bar{N}(r, \infty ; \Phi)+S(r, \Phi)
\end{gathered}
$$

Lemma 4.8. [2]. Let $\xi$ be a transcendental meromorphic function of hyper order $\rho_{2}(\xi)<1$ and $\phi(z)=\prod_{j=1}^{\eta} \xi\left(z+\omega_{j}\right)^{\mu_{j}}$, where $n, \eta, \mu_{j}(j=1,2, \ldots, \eta), \lambda=\sum_{j=1}^{\eta} \mu_{j}$ are positive integers and $\omega_{j} \in \boldsymbol{C}-\{0\}(j=1,2, \ldots, \eta)$ be distinct constants. Then

$$
(n-\lambda) T(r, \xi)+S(r, \xi) \leq T\left(r, \xi^{n} \phi\right) \leq(n+\lambda) T(r, \xi)+S(r, \xi)
$$

Lemma 4.9. [13]. Let $\xi$ be a nonconstant meromorphic function and let $l$ be a positive integer. If $\rho$ be a small function of $\xi$ then

$$
T(r, \xi) \leq \bar{N}(r, \infty ; \xi)+N(r, 0 ; \xi)+N\left(r, \rho, \xi^{(l)}\right)-N\left(r, 0,\left(\frac{\xi^{(l)}}{\rho}\right)^{\prime}\right)+S(r, \xi) .
$$

Lemma 4.10. Let $L$ be a nonconstant $L$-function and $\xi$ be a meromorphic function. Let $\tau, n, \eta, \mu_{j}(j=1,2, \ldots, \eta), \lambda=\sum_{j=1}^{\eta} \mu_{j}$ be positive integers such that $n>$ $\lambda+(\eta+1)(\tau+1)$. and $\omega_{j} \in \boldsymbol{C}-\{0\}(j=1,2, \ldots, \eta)$ be distinct constants. Also let $\rho_{2}(L)<1, \rho_{2}(\xi)<1,\left[L^{n}(z) \prod_{j=1}^{\eta} L\left(z+\omega_{j}\right)^{\mu_{j}}\right]^{(\tau)}$ and $\left[\xi^{n}(z) \prod_{j=1}^{\eta} \xi\left(z+\omega_{j}\right)^{\mu_{j}}\right]^{(\tau)}$ share $(\rho(z), l)$ and $\xi, L$ share $(\infty, 0)$, where $2 \leq l<\infty$ and $\rho(z)$ is a small function of $L$, then $L$ and $\xi$ are transcendental meromorphic functions.

Proof. By lemma 4.1 we have $T(r, L)=\frac{q}{\pi} r \log r+O(r)$, where $L$ be an L-function with degree $q$. Hence $L$ is a transcendental meromorphic function. Let $\phi(z)=$ $\prod_{j=1}^{\eta} \xi\left(z+\omega_{j}\right)^{\mu_{j}}$ and $\psi(z)=\prod_{j=1}^{\eta} L\left(z+\omega_{j}\right)^{\mu_{j}}$ Since $\left[L^{n}(z) \prod_{j=1}^{\eta} L\left(z+\omega_{j}\right)^{\mu_{j}}\right]^{(\tau)}$ and $\left[\xi^{n}(z) \prod_{j=1}^{\eta} \xi\left(z+\omega_{j}\right)^{\mu_{j}}\right]^{(\tau)}$ share $(\rho(z), l)$ therefore by lemma 4.2, lemma 4.3, lemma 
4.4, lemma 4.5, lemma 4.7 and lemma 4.9 we have

$$
\begin{aligned}
T\left(r, L^{n} \psi\right) & \leq \bar{N}\left(r, \infty ; L^{n} \psi\right)+N\left(r, 0 ; L^{n} \psi\right)+N\left(r, z ;\left(L^{n} \psi\right)^{(\tau)}\right) \\
& -N\left(r, 0 ;\left(\frac{\left(L^{n} \psi\right)^{(\tau)}}{\rho}\right)^{\prime}\right)+S(r, L) \\
& \leq(\tau+1) \bar{N}\left(r, 0 ; L^{n} \psi\right)+\bar{N}\left(r, 0 ; \frac{\left(L^{n} \psi\right)^{(\tau)}}{\rho}-1\right) \\
& -N_{\otimes}\left(r, 0 ;\left(\frac{\left(L^{n} \psi\right)^{(\tau)}}{\rho}\right)^{\prime}\right)+S(r, L) \\
& \leq(\tau+1)[T(r, L)+\eta T(r, L)]+\bar{N}\left(r, 0 ; \frac{\left(\xi^{n} \phi\right)^{(\tau)}}{\rho}-1\right)+S(r, L) \\
& \leq(\tau+1)(1+\eta) T(r, L)+T\left(r,\left(\xi^{n} \phi\right)^{(\tau)}\right)+S(r, L)
\end{aligned}
$$

By lemma 4.8 we have from (4.1)

$$
(n-\lambda) T(r, L) \leq(\tau+1)(1+\eta) T(r, L)+T\left(r,\left(\xi^{n} \phi\right)^{(\tau)}\right)+S(r, L)
$$

From (4.2) we have

$$
(n-\lambda-(\tau+1)(1+\eta)) T(r, L) \leq T\left(r,\left(\xi^{n} \phi\right)^{(\tau)}\right)+S(r, L)
$$

From (4.3) it is clear that $\xi$ is a transcendental meromorphic function since $n>$ $\lambda+(\tau+1)(1+\eta)$ and $L$ is a transcendental meromorphic function. This completes the proof of the lemma.

\section{PROOF OF THE MAIN RESULTS}

\section{Proof of Theorem 3.1}

Let $\phi(z)=\prod_{j=1}^{\eta} \xi\left(z+\omega_{j}\right)^{\mu_{j}}, \psi(z)=\prod_{j=1}^{\eta} L\left(z+\omega_{j}\right)^{\mu_{j}}, \Phi=\frac{\left(\xi^{n} \phi\right)^{(\tau)}}{\rho}$ and $\Psi=\frac{\left(L^{n} \psi\right)^{(\tau)}}{\rho}$. Then $\Phi, \Psi$ share $(1, l), 2 \leq l<\infty$ and $\Phi, \Psi$ share $(\infty, 0)$ except for zeros and poles of $\rho(z)$.

By lemma $4.10 \xi$ and $L$ are transcendental meromorphic functions.

We have by lemma 4.7 and lemma 4.8

$$
\begin{aligned}
N_{2}(r, 0 ; \Phi) & \leq N_{2}\left(r, 0 ;\left(\xi^{n} \phi\right)^{(\tau)}\right)+S(r, \xi) \\
& \leq T\left(r,\left(\xi^{n} \phi\right)^{(\tau)}\right)-T\left(r, \xi^{n} \phi\right)+N_{2+\tau}\left(r, 0 ; \xi^{n} \phi\right)+S(r, \xi) \\
& \leq T\left(r, \frac{\left(\xi^{n} \phi\right)^{(\tau)}}{\rho}\right)-(n-\lambda) T(r, \xi)+N_{2+\tau}\left(r, 0 ; \xi^{n} \phi\right)+S(r, \xi)
\end{aligned}
$$

Hence from (5.1) we have

$$
(n-\lambda) T(r, \xi) \leq T(r, \Phi)-N_{2}(r, 0 ; \Phi)+N_{2+\tau}\left(r, 0 ; \xi^{n} \phi\right)+S(r, \xi)
$$


Similarly we have

$$
(n-\lambda) T(r, L) \leq T(r, \Psi)-N_{2}(r, 0 ; \Psi)+N_{2+\tau}\left(r, 0 ; L^{n} \psi\right)+S(r, L)
$$

Now we have to consider the following two cases

Case 1 Let $\Omega \not \equiv 0$.

Using lemma 4.2, lemma 4.3 and lemma 4.6 we get from (5.2)

$$
\begin{aligned}
(n-\lambda) T(r, \xi) & \leq N_{2}(r, 0 ; \Psi)+\bar{N}(r, \infty ; \Phi)+\bar{N}(r, \infty ; \Psi) \\
& +\bar{N}_{*}(r, \infty ; \Phi, \Psi)-m(r, 1 ; \Psi)-N_{E}(r, 1 ; \Phi, \Psi \mid>3) \\
& -\bar{N}(r, 1 ; \Psi \mid>\Phi)+N_{2+\tau}\left(r, 0 ; \xi^{n} \phi\right)+S(r, \xi)+S(r, L) \\
& \leq N_{2}\left(r, 0 ;\left(L^{n} \psi\right)^{(\tau)}\right)+N_{2+\tau}\left(r, 0 ; \xi^{n} \phi\right)+S(r, \xi)+S(r, L) \\
& \leq(2+\tau)(1+\eta) T(r, L)+(\tau+2)(\eta+1) T(r, \xi) \\
& +S(r, \xi)+S(r, L)
\end{aligned}
$$

Similarly we have by lemma 4.3 and lemma 4.6 from (5.3)

$$
\begin{aligned}
(n-\lambda) T(r, L) & \leq(2+\tau)(1+\eta) T(r, \xi)+(\tau+2)(\eta+1) T(r, L) \\
& +S(r, \xi)+S(r, L)
\end{aligned}
$$

Using (5.4) and (5.5) we get

$$
\begin{aligned}
(n-\lambda)\{T(r, L)+T(r, \xi)\} & \leq(4+2 \tau)(1+\eta)\{T(r, L)+T(r, \xi)\} \\
& +S(r, \xi)+S(r, L)
\end{aligned}
$$

Hence from (5.6) we arrive at a contradiction since $n>\lambda+(4+2 \tau)(1+\eta)$.

Case 2 Let $\Omega \equiv 0$. Then $\left(\frac{\Phi^{\prime \prime}}{\Phi^{\prime}}-\frac{2 \Phi^{\prime}}{\Phi-1}\right)-\left(\frac{\Psi^{\prime \prime}}{\Psi^{\prime}}-\frac{2 \Psi^{\prime}}{\Psi-1}\right) \equiv 0$.

Integrating we have

$$
\Phi-1 \equiv \frac{\Psi-1}{C-D(\Psi-1)}
$$

where $C(\neq 0)$ and $D$ are constants.

Now we have to consider the following three cases

Subcase 2.1 Let $D=0$. Then from (5.7) we have

$$
\Phi-1 \equiv \frac{(\Psi-1)}{C},
$$


If $C \neq 1$, then from (5.8)

$$
\bar{N}(r, 0 ; \Phi)=\bar{N}(r, 1-C ; \Psi)
$$

By lemma 4.2, lemma 4.7 and the second fundamental theorem we have from (5.3)

$$
\begin{aligned}
(n-\lambda) T(r, L) & =T(r, \Psi)-N_{2}(r, 0 ; \Psi)+N_{\tau+2}\left(r, 0 ; L^{n} \psi\right)+S(r, L) \\
& \leq \bar{N}(r, 0 ; \Psi)+\bar{N}(r, 1-C ; \Psi)+\bar{N}(r, \infty ; \Psi) \\
& -N_{2}(r, 0 ; \Psi)+N_{\tau+2}\left(r, 0 ; L^{n} \psi\right)+S(r, L) \\
& \leq \bar{N}(r, 0 ; \Psi)+\bar{N}(r, 0 ; \Phi)-N_{2}(r, 0 ; \Psi) \\
& +N_{\tau+2}\left(r, 0 ; L^{n} \psi\right)+S(r, L) \\
& \leq \bar{N}\left(r, 0 ;\left(\xi^{n} \phi\right)^{(\tau)}\right)+\bar{N}\left(r, 0 ;\left(L^{n} \psi\right)^{(\tau)}\right)+N_{\tau+2}\left(r, 0 ; L^{n} \psi\right)+S(r, L) \\
& \leq N_{\tau+1}\left(r, 0 ; \xi^{n} \phi\right)+N_{\tau+1}\left(r, 0 ; L^{n} \psi\right)+N_{\tau+2}\left(r, 0 ; L^{n} \psi\right)+S(r, L) \\
& \leq(\tau+1)(\eta+1) T(r, L)+(\tau+1)(\eta+1) T(r, \xi) \\
& +(\tau+2)(\eta+1) T(r, L)+S(r, L) \\
& \leq(2 \tau+3)(\eta+1) T(r, L)+(\tau+1)(\eta+1) T(r, \xi) \\
& +S(r, L)+S(r, \xi)
\end{aligned}
$$

Similarly we have from (5.2)

$$
\begin{aligned}
(n-\lambda) T(r, \xi) & \leq(2 \tau+3)(\eta+1) T(r, \xi)+(\tau+1)(\eta+1) T(r, L) \\
& +S(r, L)+S(r, \xi)
\end{aligned}
$$

From (5.10) and (5.11) we have

$$
\begin{aligned}
(n-\lambda)(T(r, L)+T(r, \xi)) & \leq(3 \tau+4)(\eta+1)(T(r, L)+T(r, \xi)) \\
+ & S(r, \xi)+S(r, L)
\end{aligned}
$$

From (5.12) we arrive at a contradiction since $n>\lambda+(3 \tau+4)(\eta+1)$.

Hence $C=1$ and therefore we get from (5.8)

$$
\left[L(z)^{n} \prod_{j=1}^{\eta} L\left(z+\omega_{j}\right)^{\mu_{j}}\right]^{(\tau)} \equiv\left[\xi(z)^{n} \prod_{j=1}^{\eta} \xi\left(z+\omega_{j}\right)^{\mu_{j}}\right]^{(\tau)}
$$

Subcase 2.2 Let $D \neq 0$ and $C=-D$.

If $D=1$, then from (5.7) we have $\Phi \Psi \equiv 1$. Hence

$$
\left[L(z)^{n} \prod_{j=1}^{\eta} L\left(z+\omega_{j}\right)^{\mu_{j}}\right]^{(\tau)}\left[\xi(z)^{n} \prod_{j=1}^{\eta} \xi\left(z+\omega_{j}\right)^{\mu_{j}}\right]^{(\tau)} \equiv \rho(z)^{2} .
$$


If $D \neq 1$, then from (5.7) we have $\frac{1}{\Phi}=\frac{-D \Psi}{(1-D) \Psi-1}$.

Hence $\bar{N}(r, 0 ; \Phi)=\bar{N}\left(r, \frac{1}{1-D} ; \Psi\right)$.

Now proceeding as in the Subcase 2.1 we arrive at a contradiction.

Subcase 2.3 Let $D \neq 0$ and $C \neq-D$.

If $D=1$, then from (5.7) we have

$$
\Phi \equiv \frac{-C}{\Psi-C-1}
$$

Since $\xi, L$ share $(\infty, 0)$ therefore by lemma 4.2 we have from $(5.13)$

$$
\begin{aligned}
\bar{N}(r, C+1 ; \Psi) & =\bar{N}(r, \infty ; \Phi) \\
& =\bar{N}(r, \infty ; \xi)+O(\log r) \\
& =S(r, L)
\end{aligned}
$$

Now proceeding as in Subcase 2.1 we arrive at a contradiction.

If $D \neq 1$, then from (5.7) we have

$$
\Phi-\left(1-\frac{1}{D}\right) \equiv \frac{-C}{D^{2}\left(\Psi-\frac{C+D}{D}\right)} .
$$

Therefore by lemma 4.3 we have

$$
\begin{aligned}
\bar{N}\left(r, \frac{C+D}{D} ; \Psi\right) & =\bar{N}(r, \infty ; \Phi) \\
& =\bar{N}(r, \infty ; \xi)+O(\log r) \\
& =S(r, L)
\end{aligned}
$$

Hence proceeding as in Subcase 2.1 we arrive at a contradiction.

This completes the proof of the theorem.

\section{Proof of Theorem 3.2}

By lemma $4.1 L$ is a transcendental meromorphic function. Hence by lemma $4.4 Q$ is a small function of $L$. Therefore by lemma $4.10 \xi$ is a transcendental meromorphic function.

So by theorem 3.1 we get the required result. 


\section{REFERENCES}

[1] Banerjee, A., Uniqueness of meromorphic functions sharing two sets with finite weight, Portugal. Math. (N.S.) 65 1(2008), 81-93.

[2] Dyavanal, R. S., Hattikal, A. M., On the uniqueness of product of difference polynomials of meromorphic functions, Konuralp J. Math. 4(2) (2016), 42-55.

[3] Hao, W. J., Chen, J. F., Uniqueness of L-functions concerning certain differential polynomials, Dis. Dyn. Nat. Soc., 2018, Art. ID 4673165, pp. 1-12.

[4] Hayman, W. K., Meromorphic Functions, The Clarendon Press, Oxford, 1964.

[5] Lahiri, I., Weighted sharing and uniqueness of meromorphic functions, Nagoya Math. J., 161, 193-206, 2001.

[6] Lahiri, I., Weighted value sharing and uniqueness of meromorphic functions, Complex Var. Theory Appl., 46, 241-253, 2001.

[7] Lahiri, I., Mandal, N., Meromorphic functions sharing a single value with unit weight, Kodai Math. J. 29, 41-50, 2006.

[8] Lahiri, I., Mandal, N., Small functions and uniqueness of meromorphic functions, J. Math. Anal. Appl. 340, 780-792, 2008.

[9] Liu, F., Li, X. M., Yi, H. X., Value distribution of L-functions concerning shared values and certain differential polynomials, Proc. Japan Acad. Ser. A, 93, 2017, 41-46.

[10] Mandal, N., Datta, N. K., Uniqueness of L-function and its certain differential monomial concerning small functions, J. Math. Comput. Sci. 10, No. 5, 2155-2163, 2020

[11] Steuding, J., Value-distribution of L-functions, Spinger, Berlin, 2007.

[12] $\mathrm{Xu}, \mathrm{X}$. Y., On the value distribution and difference polynomials of meromorphic functions, Adv. Diff. Equ. Vol: 90 No.1 (2013), 13 Pages.

[13] Yang, L., Normality for families of meromorphic functions, Sci. Sinica Ser. A 29, no. 12, 1263-1274, 1986. 
[14] Yang, C. C., Yi, H. X., Uniqueness Theory of Meromorphic Functions, Kluwer Academic Publishers, Dordrecht, 2003; Chinese original: Science Press, Beijing, 1995.

[15] Yang, L., Value distribution theory, Spinger Verlag Berlin, 1993.

[16] Zhu, W. Q., Chen, J. F., Differential polynomials of L-functions with truncated shared values, Open Math. 2019; 17, 1113-1125. 
